\title{
Neutron Scattering Studies on the Crystallization of Polymers
}

\author{
E. W. FISCHER \\ Institut für Physikalische Chemie der Universität Mainz, \\ 6500 Mainz, FRG
}

(Received August 20, 1984)

\begin{abstract}
The morphology of semicrystalline polymers can be described to a good approximation as consisting of lamellar crystallites separated by amorphous regions. The way in which a single macromolecule traverses the crystalline and amorphous phases can be evaluated from neutron scattering studies of mixtures of deuterated and protonated samples of the same polymers. A new method is proposed for the evaluation of the neutron scattering data without introducing detailed structural models. The only assumption made is that the molecular structure can be described as consisting of "clusters" of crystalline stems which belong to the same molecule. It is shown that this cluster model can be verified experimentally for the cases of poly(ethylene oxide), polypropylene and polyethylene. The spatial correlation of the crystalline stems within a cluster can be evaluated from the scattering data by introducing a direct correlation function $c(x)$. The method was checked by Monte-Carlo calculations and applied to the neutron scattering data of poly(ethylene oxide) (PEO) in the wide angle range. The fraction of stems occupying adjacent sites in the crystal lattice depends strongly on crystallization conditions.

KEY WORDS Crystallization / Neutron Scattering / Monte-Carlo

Calculations / Poly(ethylene Oxide) /
\end{abstract}

Semicrystalline polymers with a high degree of crystallinity generally consist of stacks of lamellar crystals separated one from another by amorphous layers. This structural model has been well established by numerous electron microscope and small angle X-ray scattering studies. As an example Figure 1 shows the electron micrograph ${ }^{1}$ of a polyethylene fraction crystallized at $T_{\mathrm{c}}=130^{\circ} \mathrm{C}$. The amorphous regions appear as black areas because of the staining with uranyl acetate. ${ }^{2}$ However, it is well established that the polymer molecule in the melt forms a coil with unperturbed dimensions. The insertion in Figure 1 shows a polyethylene molecule with $M=10^{5}$ generated by the Monte-Carlo meth$\operatorname{od}^{3}$ and the approximate volume of a single molecule $\left(R_{\mathrm{g}} \approx 184 \AA\right)$ is also indicated in the scale of the electron micrograph. The problem arises what is the conformation of the molecule in the crystalline state and how does it traverse the crystalline and amorphous regions. Various models have been proposed ${ }^{4}$ and Figure 2 shows the schematic diagrams of two examples. ${ }^{5,3 \mathrm{a}}$
The models differ especially with regard to the assumptions made about the nature of the so-called fold surface and about the number of "re-entries" of a chain molecule into the same lamella. The structure of the amorphous regions has important implications, some of them are listed in Table I. There are several other reasons why we want to know the trajectory of a single chain in the semicrystalline polymer, as seen in Table II.

It is quite obvious that the molecular conformation is governed by all the factors which effect the crystalline-amorphous texture or morphology. In Figures 1 and 3 the influence of the molecular mass $M_{w}$ is demonstrated. With increasing $M_{w}$ the regularity of the morphological pattern decreases as one would naively expect. Figure $3 \mathrm{c}$ shows the effect of crystallization temperature (compare with Figure 1). One must assume that the chain conformation is correlated to those variations of morphology. Therefore it is not enough to prove or to disprove certain models, but one looks for a method which enables us to determine the relevant structure pa- 


\section{E. W. FISCHER}

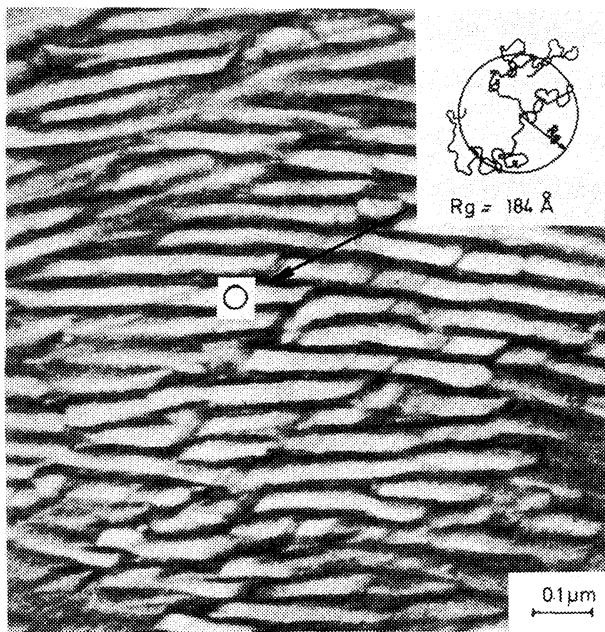

Figure 1. Electron micrograph of an ultrathin section of stained polyethylene, $M_{\mathrm{w}}=189,000$, crystallized at $T_{\mathrm{c}}=130^{\circ} \mathrm{C} .^{1}$ The insertion shows a polyethylene molecule generated by the Monte-Carlo method, ${ }^{3}$ and a reduction to the scale of the micrograph $\left(R_{\mathrm{g}}\right.$ in the melt $\sim 184 \AA$ ).

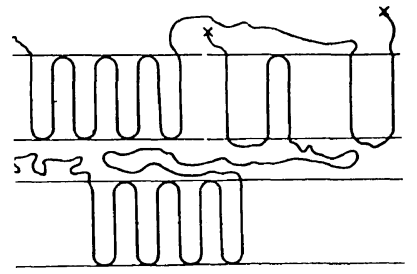

( A )

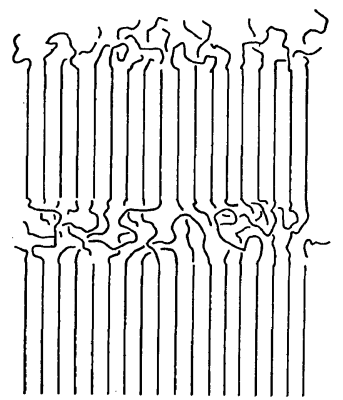

(B)

Figure 2. Two examples of proposed models for the structure of the amorphous regions in semicrystalline polymers. (A) Central core model with 8 adjacent reentry folds in the cores, ${ }^{5}$ (B) "Switchboard" model. ${ }^{3 a}$

rameters in a quantitative manner.

Direct information about the chain conformation can be obtained from neutron scattering studies of
Table I. Nature of the amorphous regions in semicrystalline polymers

\begin{tabular}{cl}
\hline \multicolumn{1}{c}{ Questions } & \multicolumn{1}{c}{ Some implications } \\
\hline $\begin{array}{c}\text { Number of tie molecules } \\
\text { and cilia }\end{array}$ & $\begin{array}{l}\text { Strength of polymers } \\
\text { Fracture, crazing and yield } \\
\text { Conformation of non- } \\
\text { crystalline sequences }\end{array}$ \\
$\begin{array}{c}\text { Deformation behaviour } \\
\text { Glass transition temperature } \\
\text { Diffusion, physical aging } \\
\text { tional order in } \\
\text { amorphous regions }\end{array}$ & Mechanical modulus \\
\hline
\end{tabular}

Table II. Why do we want to know the single chain trajectory?

1) Theory of cyrstallization

Dependence of structure and crystallization rate on molecular mass, crystallization temperature $T_{\mathrm{c}}$ and chemical defects (copolymers)

Role of entanglements

Reasons for "bad" and "good" crystallization behaviour

Crystallization under extensional flow

2) Further implications

Disordered regions in high-modulus fibers

Conformation of soft segments in block-

copolymers

Transition of stiff chains from the liquid-crystalline state into the crystalline state

mixtures consisting of deuterated polymers and cocrystallized with protonated chains of the same polymer. This technique allows the evaluation of the single chain structure factor $P(q)$, where $q=$ $(4 \pi / \lambda) \sin \theta / 2$. The main aim of the following paper is to show what kind of information can be obtained from the various ranges of the scattering vector $q$ without detailed assumptions about the structure. So we ask ourselves how far a straight-forward evaluation of the scattering data will yield relevant structure parameters. The proposed methods are applied to poly(ethylene oxide), polyethylene and polypropylene, and the results are discussed which were obtained so far.

\section{THE SMALL ANGLE RANGE}

The principle of the neutron scattering technique is well known. ${ }^{6}$ After a correct subtraction of the 


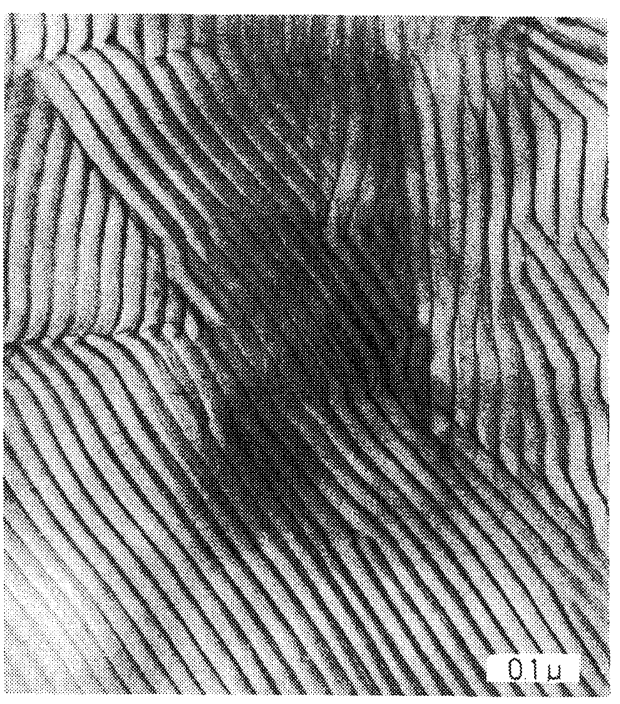

(a)

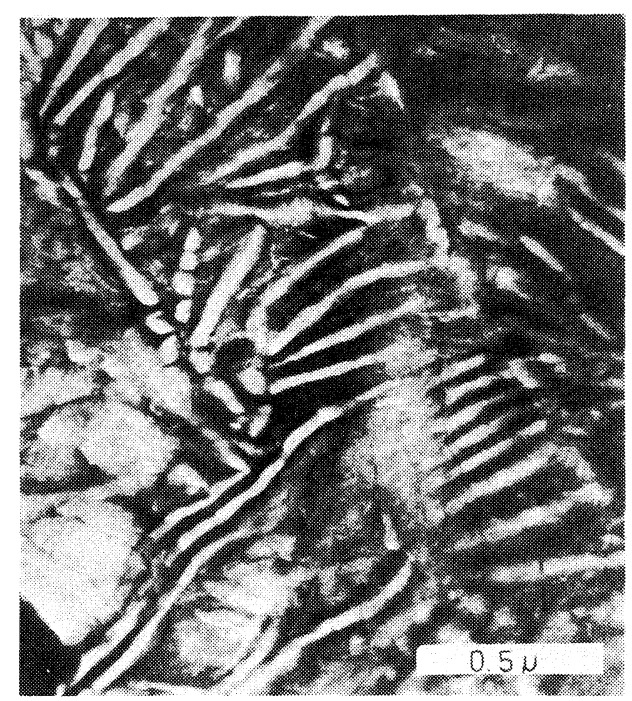

(b)

incoherent "background" and the contributions due to density fluctuations, the differential scattering cross section per unit volume $\mathrm{d} \sigma / \mathrm{d} \Omega$ is given by

$$
\frac{\mathrm{d} \sigma}{\mathrm{d} \Omega}(q)=c_{\mathrm{D}}\left(1-c_{\mathrm{D}}\right) K n_{\mathrm{w}} P(q)
$$

provided that there exists no thermodynamic interaction between $\mathrm{H} / \mathrm{H}$ and $\mathrm{D} / \mathrm{D}$ molecules (no segregation or intermolecular clustering). In eq $1 c_{\mathrm{D}}$ is the concentration of deuterated molecules, $K$ is

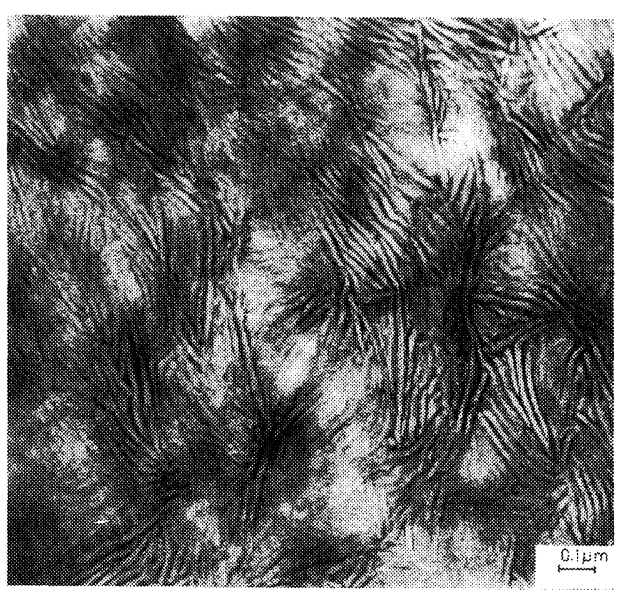

(c)

Figure 3. Electron micrographs of polyethylene crystallized from the melt, ${ }^{1}$ (a) $M_{\mathrm{w}}=5600, T_{\mathrm{c}}=127^{\circ} \mathrm{C}$, (b) $M_{\mathrm{w}}=1.6 \times 10^{6}, T_{\mathrm{c}}=130^{\circ} \mathrm{C}$, (c) $M_{\mathrm{w}}=189,000, T_{\mathrm{c}}=116^{\circ} \mathrm{C}$ (for comparison with Fig. 1).

the contrast factor, $n_{\mathrm{w}}$ the degree of polymerization, and $P$ the form factor of the polymer molecule:

$$
P(q)=\frac{1}{n_{\mathrm{w}}{ }^{2}}\left\langle\sum_{i, j} \exp \left[i q\left(R_{i}-R_{j}\right)\right]\right\rangle
$$

which may be approximated for small $q$ by

$$
P(q) \approx \exp \left(-\frac{1}{3} q^{2} R_{\mathrm{g}}^{2}\right)
$$

or by

$$
1 / P(q) \approx 1+\frac{1}{3} q^{2} R_{\mathrm{g}}^{2}
$$

where $R_{\mathrm{g}}$ is the radius of gyration.

For the evaluation of the data it is useful to introduce the reduced scattering intensity

$$
J(q)=\frac{\mathrm{d} \sigma / \mathrm{d} \Omega}{c_{\mathrm{D}}\left(1-c_{\mathrm{D}}\right) K}=n_{\mathrm{w}} P(q)
$$

or a "scattering function"

$$
F_{\mathrm{n}}(q)=n_{\mathrm{w}} P(q) q^{2}
$$

This function is very often used for comparison of measured and calculated data.

In Table III the various information is summarized $^{7,8}$ which can be obtained in three different $q$-ranges characterized very roughly by the numbers given. In the small angle range (SANS) the radius of 
Table III. Information obtainable from neutron scattering experiments in various angular ranges

\begin{tabular}{cl}
\hline Range & \multicolumn{1}{c}{ Informations } \\
\hline SANS \\
$0.005<q / \AA^{-1}<0.03$ & $\begin{array}{l}\text { Molecular weight } \\
\text { Radius of gyration } R_{\mathrm{g}} \\
\text { Segregation effects }\end{array}$ \\
\hline IANS & $\begin{array}{l}\text { Average number of stems per } \\
\text { "cluster" in one lamella } \\
\text { Average distance between } \\
\text { crystalline stems of the } \\
\text { same moelcule in a lamella }\end{array}$ \\
\hline WANS & $\begin{array}{l}\text { Direct correlation function } \\
\text { of stems }\end{array}$ \\
\hline $0.3<q / \AA^{-1}<5$ &
\end{tabular}

gyration $R_{\mathrm{g}}$ of the molecules in the semicrystalline state can be measured. The apparent molecular weight obtained by extrapolation $q \rightarrow 0$ can be used for control of segregation effects. In the intermediate angle range (IANS) integral informations is obtained about the spatial correlation of crystalline stems. As will be shown later, mainly the average density of stems and the number of stems per "cluster" can be measured. Most valuable results have to be expected from the upper range of IANS and from the wide angle scattering (WANS), that means in the range of about $0.2<q<2 \AA^{-1}$. The reasons are quite obvious if one takes into account that the various structure models differ with regard to the spatial correlation of the crystalline stems of one and the same molecule in the range of 5 to $20 \AA$, let's say.

We first discuss the results obtained in the small angle range. For $q R_{\mathrm{g}}<1$ the scattering is due to the overall mass distribution of a polymer chain and yields valuable information about the "dilution" of a single chain in the crystalline state. The radius of gyration $R_{\mathrm{g}}$ can be evaluated from eq 4 without any specific model assumptions. On the other hand only this one integral number can be obtained from SANS measurements and therefore it is quite obvious that this $q$-range is not suitable for deciding between different structure models.

The evaluation of $R_{\mathrm{g}}$ may be distorted by phase separation between $\mathrm{H}$ - and D-molecules which frequently occurs during crystallization. In so far as reliable information could be obtained, it turned

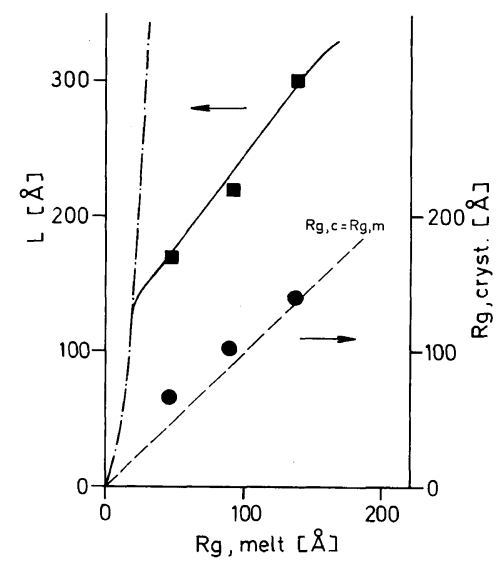

Figure 4. The long spacing $L$ and the radius of gyration $R_{\mathrm{g}, \text { cryst }}$ of polyethylene oxide quickly crystallized by quenching to $T_{\mathrm{c}}=40^{\circ} \mathrm{C}$ in dependence on the radius of gyration $R_{\mathrm{g} \text {, melt }}$ in the melt. $R_{\mathrm{g}}$ values are weightaverages. The broken line gives approximate values $L$ for extended chain crystals.

out that the gyration radius in the crystalline state often does not differ markedly from that in the melt or in dilute solution. This was found by Schelten $e t$ $a l{ }^{9}$ for melt-quenched linear polyethylene and by Ballard et al: ${ }^{10}$ for isotactic polypropylene crystallized and annealed under various conditions. For isotactic polystyrene crystallized near its melting point a pronounced increase of $R_{\mathrm{g}}$ was reported. ${ }^{11}$ Since the majority of those values were evaluated far from the Guinier range and since these samples had a rather low crystallinity $(\sim 40 \%)$ it is not quite clear at the moment, whether the reported effect can be generalized. In the case of poly(ethylene oxide) quickly crystallized by quenching to $T_{\mathrm{c}}=40^{\circ} \mathrm{C}$ only slight deviations of the radius of gyration $R_{\mathrm{g}}$ (cryst) from the value $R_{\mathrm{g}}$ (melt) have been found ${ }^{12}$ as shown in Figure 4.

In this connection it may be interesting to notice that the long spacing $L$ of the crystallized samples as measured by SANS and small angle X-ray scattering strongly increases with molecular mass $M_{w}$, as also shown in Figure 4. This observation cannot be explained on the base of the conventional crystallization theory. ${ }^{13}$ If the crystal thickness would be determined by the supercooling $\Delta T=T_{\mathrm{m}}^{0}-T_{\mathrm{c}}$ one should expect a slight decrease with increasing $M_{w}$. We will discuss the long spacing again in connection with the results obtained from the intermediate $q$ range. 

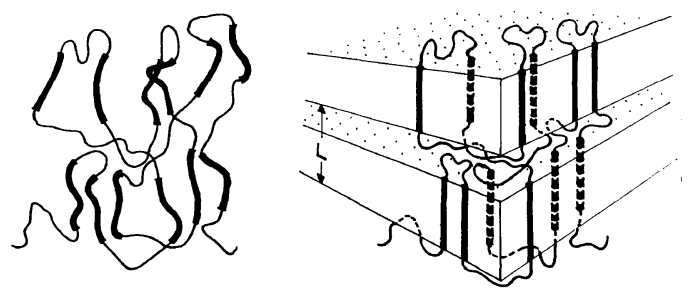

Figure 5. "Erstarrungsmodell" (solidification model) of the crystallization process of chain molecules. The fully drawn sequences of the coil are incorporated into the growing lamellae without long range diffusion or major reorganization of the chain conformation. ${ }^{7,8,14}$ Note that the dimensions are not drawn in right scale. The length of the crystalline stems is much larger than their mutual distances.

If we restrict our considerations to crystallization conditions where $R_{\mathrm{g}}$ remains approximately invariant during crystallization, the most simple explanation may be based on a model which we call "Erstarrungsmodell" (solidification model). ${ }^{7,8,14}$ In this rather naive picture, see Figure 5, it is assumed that the crystallization occurs only by straightening of suitably oriented sequences of the coil which are incorporated into the crystalline lamella. This should happen in such a way that the "volume" occupied by that part of the chain which is incorporated into one lamella is not changed appreciably during crystallization. The main point of this model is that no long range diffusional process will take place and that the "dilution" of the considered chain by other chains remains constant. The model also implies that the number of entanglements which are present in the melt has not to be changed, they are just shifted to the amorphous regions. The development of long crystalline "stems" necessarily causes the average distance between the centers of the stems to be much smaller than the distance of the same chain units before crystallization. Otherwise $R_{\mathrm{g}}$ could not be kept constant. In so far Figure 5 is not right in scale and has provoked a criticism, ${ }^{15}$ which was based on the assumption of a two-dimensional projection of the initial random walk in the melt (see Figure 1 of ref 15). It has been demonstrated that the results of such a treatment do not agree with the experimental data. As we have shown earlier, ${ }^{16}$ however, the distance distribution of the crystalline stems is narrower than expected on the basis of this model.

Since only one integral quantity - the radius of gyration $R_{\mathrm{g}}-$ can be evaluated from the small angle region, this range is of a rather limited use for our main goal to learn something about the chain conformation in the crystalline state.

\section{THE INTERMEDIATE $q$-RANGE}

From the intermediate $q$-range $0.03<q / \AA^{-1}<0.3$ one may expect to obtain more detailed information about the spatial correlations of the crystalline stems belonging to the same molecule. One useful way for evaluating the experimental data is the computation of the scattering function for certain models. $^{3,4,3 a, 8,17}$ The calculated function can be compared with the experimental results. So for example Figure 6 shows a distance distribution of successive stems resulting from Monte-Carlo calculations for the case of quenched polyethylene. ${ }^{8,16}$ The distribution was adjusted in the way that the best fit with experimental data was obtained.

It turned out, however, that satisfactory agreement can be reached on the basis of quite different models. ${ }^{4}$ Therefore we are now concerned with the question which information can be obtained from the intermediate $q$-range in a straight-forward manner without the introduction of detailed structural models. It is assumed only that the crystalline stems are incorporated in crystalline lamellae, see Figure 7 , and that the distances between stem centers within one package or "cluster" of stems located in one lamella are much smaller than the distances between stem centers belonging to different lamellae. Details of the chain re-entry or chain tilting are not taken into account at the moment. We assume further that the sample has a high degree of crystallinity $w_{\mathrm{c}}$ and that a well pronounced long spacing reflection is observed by SAXS.

As a consequence of a structure as pictured in Figure 7 the clusters of stems in one lamella will scatter as independent units in a certain intermediate $q$-range. Only at very small $q$ the interconnection of the clusters by means of the tie molecules will be reflected in the scattering curve. We now discuss the scattering due to the uncorrelated clusters.

In order to simplify the treatment we consider a molecular consisting of $v$ clusters each of $N_{\mathrm{c}}$ stems, so that

$$
w_{\mathrm{c}} n_{\mathrm{w}}=v N_{\mathrm{c}} n_{\mathrm{st}}
$$




\section{E. W. FISCHER}

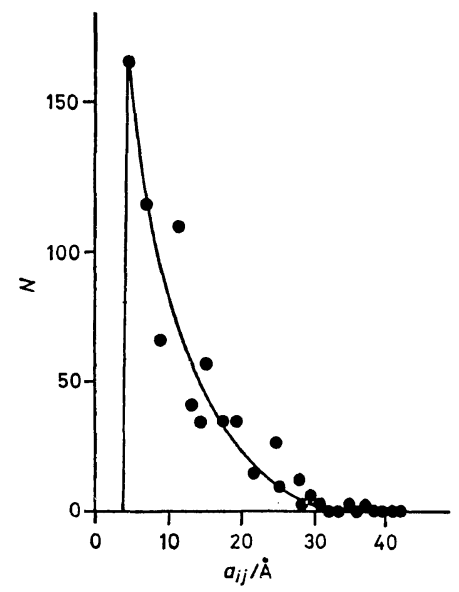

Figure 6. Evaluation of neutron scattering data of quenched polyethylene by means of Monte-Carlo calculations. ${ }^{8,16}$ The shown distribution of the distances between successive stems yields a good fit with experimental results.

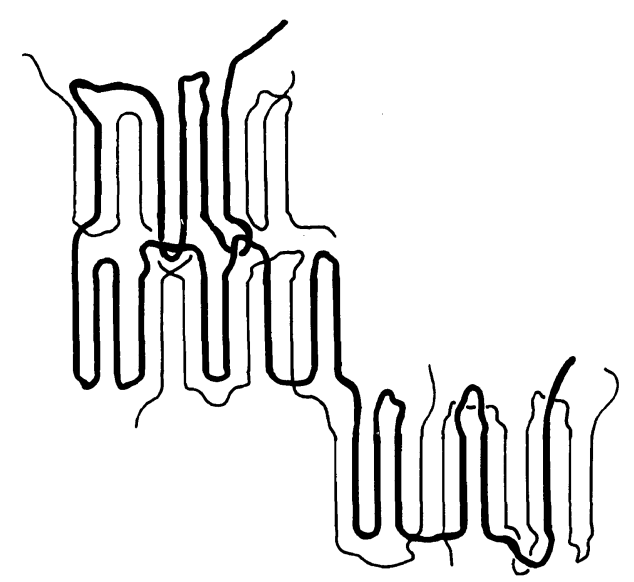

Figure 7. Schematic representation of a single macromolecule transversing different lamellar crystals. Clusters of crystalline stems situated in different lamellae are connected by tie molecules. Note that the drawing is not in correct scale: The length of the crystalline stems is about 50 times larger than their lateral distances.

where $n_{\mathrm{st}}$ is the number of monomer units per stem. The effects of a distribution of cluster sizes $N_{\mathrm{c}}$ has been considered in great detail in a recent publication. ${ }^{18}$ The reduced scattering intensity of a single cluster is given by

$$
J(q)=n_{\mathrm{st}} P_{\mathrm{s}}(q) \frac{1}{N_{\mathrm{c}}} \sum_{m, n}^{N \mathrm{c}} J_{0}\left(q x_{m n}\right)
$$

where $J_{0}$ is the Besselfunction of order zero, which appears as a consequence of the fact that all stems in one cluster are parallel to each other. $x_{m n}$ is the distance between the $m$-th and $n$-th stems of the cluster. $P_{\mathrm{s}}(q)$ means the formfactor of the stem, which can be easily calculated from the crystallographic data of the unit cell.

The scattering of the uncorrelated clusters depends only on the number $N_{\mathrm{c}}$ of stems per cluster and on the mutual arrangement of the stems. We therefore introduce a stem correlation function

$$
H(q)=\frac{1}{N_{\mathrm{c}}} \sum_{m \neq n}^{N_{\mathrm{c}}}\left\langle J_{0}\left(q x_{m n}\right)\right\rangle
$$

and consequently one obtains

$$
J(q)=n_{\mathrm{st}} P_{\mathrm{s}}(q)(1+H(q))
$$

The properties of $H(q)$ for large $q$-values $\left(q>0.2 \AA^{-1}\right)$ depend strongly on the direct correlation between subsequent stems which are connected by a loop or fold. This interaction can be described by a direct correlation function $c(x)$, which we will discuss in the next paragraph. At the moment we are only interested in the intermediate $q$-range, let us say $0.03<q / \AA^{-1}<0.2$. Without any special assumptions about $H(q)$ one obtains

$$
H(q)=\frac{1}{N_{\mathrm{c}}} \sum_{m \neq n}^{N_{c}}\left(1-\frac{\left\langle\left(q x_{m n}\right)^{2}\right\rangle}{4}+\cdots\right)
$$

or

$$
H(q) \approx\left(N_{\mathrm{c}}-1\right)-\frac{N_{\mathrm{c}}}{2} R_{\mathrm{cc}}^{2} q^{2}
$$

with the radius of gyration of the stem centers in a cluster

$$
R_{\mathrm{cc}}^{2}=\frac{1}{N_{\mathrm{c}}} \sum_{n}^{N \mathrm{c}} x_{n, 0}^{2}
$$

where $x_{n, 0}$ is the vector from the center of gravity of the cluster to the $n$th center of a stem.

Using this approximation the number $N_{\mathrm{c}}$ of stems per cluster and the dimensions of the clusters can be determined easily. By means of eq 10 the values $H(q)$ can be calculated from the measured data $J(q)$ and they are plotted in the usual way of $H^{-1}$ versus $q^{2}$ :

$$
H(q)^{-1} \approx \frac{1}{N_{\mathrm{c}}-1}\left(1+\frac{N_{\mathrm{c}}}{2\left(N_{\mathrm{c}}-1\right)} R_{\mathrm{cc}}^{2} q^{2}\right)
$$

The intersection and the slope will yield the desired 


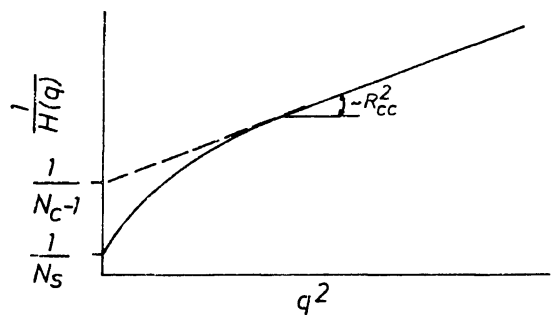

Figure 8. Schematic drawing of the stem correlation function $H(q)$ in the Zimm plot $H(q)^{-1}$ vs. $q^{2}$. Extrapolation $q \rightarrow 0$ yields the average number $N_{\mathrm{c}}$ of stems per cluster.

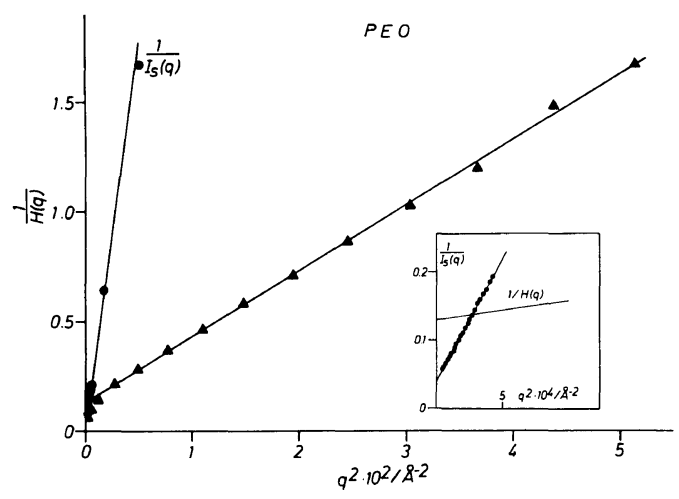

Figure 9. Evaluation of $\left\langle N_{\mathrm{c}}\right\rangle$ and $\left\langle R_{\mathrm{cc}}^{2}\right\rangle$ from $H(q)^{-1}$ $v s . q^{2}$ for poly(ethylene oxide) quickly crystallized by quenching to $T_{\mathrm{c}}=40^{\circ} \mathrm{C}$. Long spacing $L=300 \AA$. The insert shows the experimental data for $J(q) v s . q^{2}$.

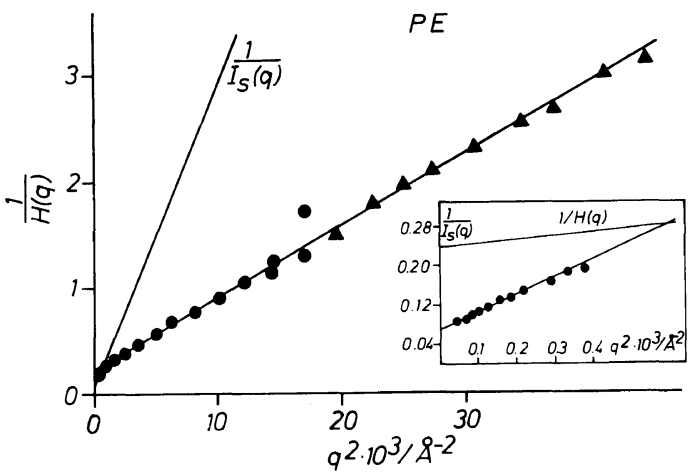

Figure 10. As in Figure 9 for polyethylene crystallized by quenching from the melt. 0 , Schelten et al., ${ }^{9} ; \mathbf{\Delta}$ Stamm et al. ${ }^{8}$

information. From $N_{\mathrm{c}}$ and the known number of all crystalline stems belonging to one molecule the average number $v$ of clusters can be determined.
The procedure is demonstrated schematically in Figure 8 . In general eq 14 will only hold within the so-called Zimm-range, i.e., for $q^{2} \cdot R_{\mathrm{cc}}^{2}<1$. Using experimental data it turned out however, that $H(q)^{-1} v s . q^{2}$ yields a straight line over a range up to $q^{2} \cdot R_{\mathrm{cc}}^{2} \sim 2$ or even larger. (See for example Figures 9 and 10.) This special property of $H(q)$ is of course determined by the character of the direct correlation function $c(x)$, as we will discuss later.

The application of the method described by Figure 8 to experimental neutron scattering data was quite successful in all cases so far studied, ${ }^{18}$ that means for poly(ethylene oxide), polyethylene, and isotactic polypropylene. For example Figure 9 shows the experimental data for PEO $\left(M_{w}=\right.$ $125,000)$ quickly crystallized by quenching to $T_{\mathrm{c}}=$ $40^{\circ} \mathrm{C}$. In Figure 10 the same procedure was applied to polyethylene crystallized by quenching. In both figures the inserts show the normalised intensity $J(q)$ for the small angle range and for comparison the lines $J(q)^{-1}$ versus $q^{2}$ are also drawn in the plots. As one can notice a clear distinction is possible between the (uncorrelated) cluster scattering in the intermediate $q$-range and the scattering by the correlated clusters in the SANS range. Similar results ${ }^{18}$ were obtained from the literature data of neutron scattering studies of isotactic polypropylene. ${ }^{19}$

Before we discuss the numerical results of this method some remarks are in order concerning the separation of the "cluster" scattering. The experimental results confirm our basic assumption about well defined clusters of crystaline stems belonging to one molecule in one lamella. In so far the model of Figure 7 is similar to the "core-model" proposed by other authors. ${ }^{17,20}$ The separation of the cluster scattering has also been described already. ${ }^{21}$ Such clusters cannot be formed if an emerging chain enters another lamella with an high probability and then re-enters the former lamella again building up a large number of tie-molecules. There are good reasons to believe that the experimentally proved existence of well defined clusters is due to the fact, that the growth fronts of the lamellae of a stack do not arrive simultaneously at the position of a macromolecule. In Figure 11 this situation is schematically demonstrated. The stepwise growth will result in approximately $(v-1)$ tie-molecules if $v$ is the average number of clusters per molecule. The growth of the cluster within one and the same lamella is supposed to be stopped by kinetic hin- 
Table IV. Results of neutron scattering studies in the intermediate $q$-range. Poly(ethylene oxide) of different molecular mass crystallized under various conditions

\begin{tabular}{|c|c|c|c|c|c|}
\hline & $\begin{array}{l}\text { PEO I } \\
\left(40^{\circ} \mathrm{C}\right)\end{array}$ & $\begin{array}{l}\text { PEO II } \\
\left(40^{\circ} \mathrm{C}\right)\end{array}$ & $\begin{array}{l}\text { PEO III } \\
\left(40^{\circ} \mathrm{C}\right)\end{array}$ & $\begin{array}{l}\text { PEO II } \\
\left(53^{\circ} \mathrm{C}\right)\end{array}$ & $\begin{array}{c}\text { PEO III } \\
\text { (slowly cooled) }\end{array}$ \\
\hline Molecular mass $M_{w}\left(\mathrm{~g} \mathrm{~mol}^{-1}\right)$ & 15,000 & 55,000 & 125,000 & 55,000 & 125,000 \\
\hline Long spacing $L(\AA)$ & 170 & 220 & 300 & 300 & 430 \\
\hline $\begin{array}{l}\text { Number of crystalline stems } N_{\mathrm{s}} \\
\text { Radius of gyration of the whole }\end{array}$ & 5.9 & 16.7 & 25.0 & 9.2 & 17.3 \\
\hline $\begin{array}{l}\text { molecule in the crystalline state } \\
\left\langle R^{2}\right\rangle_{z}^{1 / 2}(\AA)\end{array}$ & 73 & 117 & 160 & 103 & 221 \\
\hline $\begin{array}{l}\text { Average number of stems } N_{\mathrm{c}} \\
\text { per cluster }\end{array}$ & 4.1 & 8.1 & 8.7 & 7.0 & 6.5 \\
\hline $\begin{array}{l}\text { Average number } v \text { of } \\
\text { clusters per molecule }\end{array}$ & 1.4 & 2.1 & 2.9 & 1.3 & 2.7 \\
\hline $\begin{array}{l}\text { Radius of gyration of the } \\
\text { stem centers } \\
\left\langle R_{\mathrm{CC}}^{2}\right\rangle_{z}^{1 / 2}(\AA)\end{array}$ & 15 & 23 & 20 & 20 & 27 \\
\hline $\begin{array}{l}\text { Average distance }\left\langle a_{1}\right\rangle(\AA) \\
\text { (random walk) }\end{array}$ & 21 & 21 & 19 & 20 & 26 \\
\hline $\begin{array}{l}\text { Average distance }\left\langle a_{2}\right\rangle(\AA) \\
\text { (linear arrangement of the stems) }\end{array}$ & 17 & 11 & 9 & 12 & 17 \\
\hline
\end{tabular}

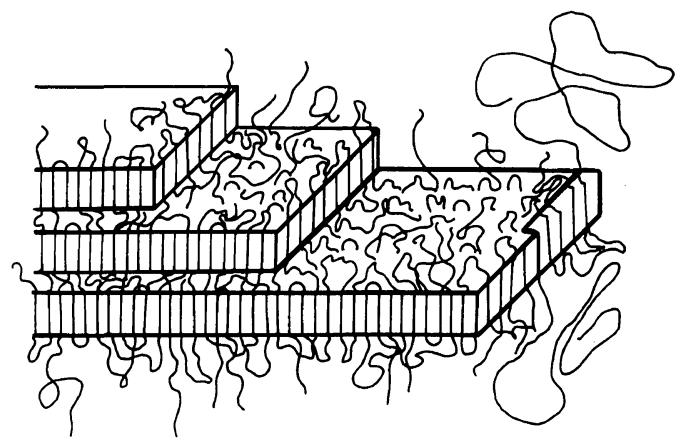

Figure 11. Schematic diagram of the growth of a stack of lamellae in the melt. The growth fronts do not arrive simultaneously at the location of a single molecule.

drance, e.g., by entanglements, and by filling up the growth front with other molecules. ${ }^{13}$

The results obtained for the case of poly(ethylene oxide $)^{12}$ are summarized in Table IV, which we will now discuss in some detail. The over-all number of crystalline stems $N_{\mathrm{s}}$, which was evaluated from plots like Figure 9, is in excellent agreement with the value expected from the long spacing $L$ and the molecular mass as measured by GPC. The $z$-average of the radius of gyration of the whole molecule is somewhat larger than the value obtained for the melt, see also Figure 4. The difference is especially large for the sample with $L=430 \AA$. Comparison of the data shows that the average number $v$ of clusters per molecule is independent of the crystallization conditions, whereas the radii of gyration of the stem centers $\left\langle R_{\mathrm{cc}}^{2}\right\rangle^{1 / 2}$ differ by roughly $25 \%$, indicating that the stem distribution within a lamella depends on the crystallization conditions.

The quantities $R_{\mathrm{cc}}^{2}$ depend on the packing densities of the stems within the clusters. For illustration the average distances $\langle a\rangle$ between the stems are given in Table IV for two extreme cases. First a random walk arrangement was assumed, on the other hand $\langle a\rangle$ was also calculated for linear arrangement of the stems by the approximation

$$
\left\langle a_{2}\right\rangle^{2}=12 R_{\mathrm{cc}}^{2} /\left(N_{\mathrm{c}}-1\right)^{2}
$$

under both assumptions the average distance is appreciably larger than the nearest neighbour distance in the PEO lattice.

Usally the scattering data in the intermediate $q$ range are plotted in the so-called Kratky representation $I(q) \cdot q^{2}$ versus $q$. For various polymers (polypropylene, poly(ethylene oxide) and isotactic polystyrene) a plateau has been observed in this representation, in the case of polyethylene a slight increase was obtained. Therefore the question arises 


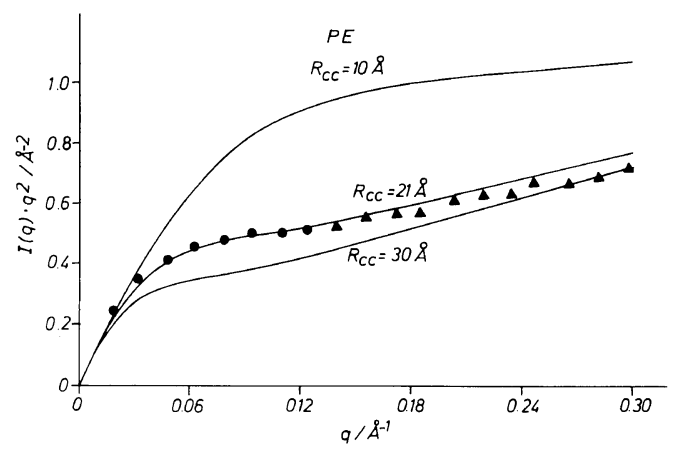

Figure 12. Scattering curves in Kratky representation calculated from eq 10 and assuming an approximation of $H(q)$ given by eq 14. Measured data for polyethylene (references as in Figure 10).

whether this experimentally observed behaviour can be approximately reproduced by the single cluster scattering described above. According to eq 10 the reduced intensity $J(q)$ in this $q$-range is determined by the single stem form factor $P_{\mathrm{s}}(q)$ and the correlation function $H(q)$ which can be approximated by eq 14 . As an example in Figure 12 the Kratky plot of experimental data of polyethylene is reproduced. The drawn curves are calculated on the basis of the assumption of eq 10 and 14 with various parameters $R_{\mathrm{cc}}$. As one can see, for a constant number $N_{\mathrm{c}}$ of stems per cluster the plateau height depends strongly on the radius of gyration $R_{\mathrm{cc}}$. As has been pointed out previously ${ }^{22}$ the height of the $q^{2}$-plateau is controlled by the average distance $\langle a\rangle$ of the stems within one cluster.

It is important to note that the analysis presented above will yield only those integral quantities such as $N_{\mathrm{c}}$ and the second moment of the correlation function $h(x)$ of the stem centers within a cluster. It is quite clear that up to a $q$-range of about $0.15-$ $0.2 \AA^{-1}$ no further information can be obtained, no matter how one plots the results. In particular it is not possible in that $q$-range to decide upon the question how the chain re-entry within one cluster occurs. The detailed character of the correlation function $h(x)$ can only be evaluated from measurements in the wide angle regime.

We close the discussion of the intermediate $q$ range with a remark on the surprising dependence of the long spacing $L$ on molecular weight. If one calculates from Table IV the molecular mass $M_{v}$ per cluster and accordingly the radius of gyration $R_{\mathrm{g}, v}$

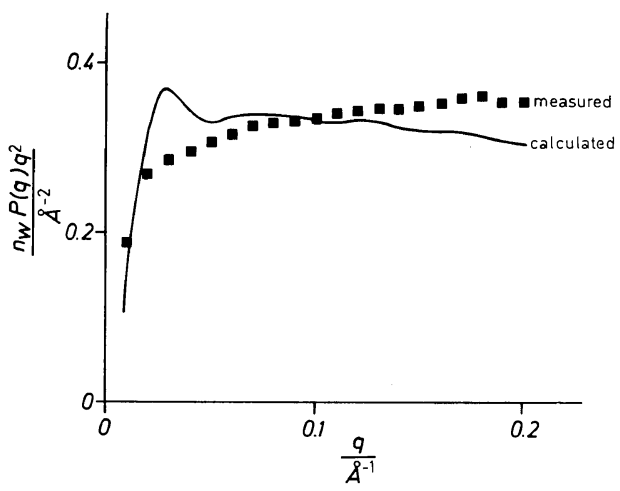

Figure 13. Scattering function $F_{\mathrm{n}}(q)=I(q) \cdot q^{2}$ for quickly crystallized PEO $\left(T_{\mathrm{c}}=40^{\circ} \mathrm{C}, M_{\mathrm{w}}=125,000\right)$ in comparison with calculated $F_{\mathrm{n}}(q)$ for adjacent re-entry along the (100) plane. Intermediate $q$-range $q \leq 0.2 \AA^{-1}$.

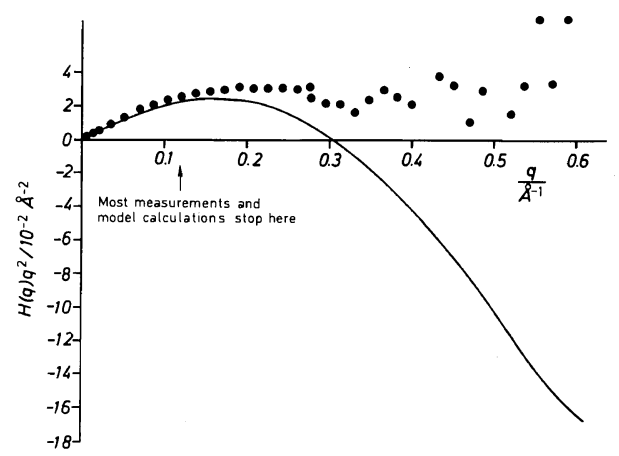

Figure 14. Comparison of measured and calculated stem correlation function $H(q) q^{2}$ for the same sample as in Figure 10, for a $q$-range up to $q=0.6 \AA^{-1}$.

of this part of the molecule in the melt one obtains in a good approximation

$$
L^{2} / 12 \approx R_{\mathrm{g}, v}^{2}
$$

which speaks again in favour of the solidification model.

\section{THE DIRECT CORRELATION FUNCTION $c(x)$}

As it has been shown above the properties of the scattering function $I(q) q^{2}$ in the intermediate $q$ range depend only on the average distance between the crystalline stems within a cluster. A distinction between various models of the crystallization process cannot be made on the base of scattering data from this range. This is demonstrated by Figures 13 
and 14. In Figure 13 a measured scattering function of PEO crystallized at $40^{\circ} \mathrm{C}$ is compared with the calculated scattering function for an adjacent reentry model under the assumption that the re-entry takes place along the (100) plane. With regard to the level of the scattering function $F_{\mathrm{n}}=n_{\mathrm{w}} P(q) q^{2}$ no strong discrepancy seems to exist within the $q$-range $q<0.2 \AA^{-1}$. If one compares the measured and calculated data for larger $q$ 's, those curves differ completely, as demonstrated by Figure 14. There the measured stem correlation function $H(q)$ is compared with the calculated correlation function for the case of adjacent re-entry. The discrepancy shows up for $q$-values $q>0.15 \AA^{-1}$ and it may be noticed that most measurements and model calculations stop at about this $q$-value so far as the data in the literature are concerned.

This example clearly demonstrates that for the evaluation of the details of the stem correlation function $h(x)$ the wide angle scattering range has to be considered. This can be done by calculating the formfactor of a molecule according eq 2 for large $q$ 's assuming certain models. For the case of polyethylene and under the assumption of adjacent reentry the wide angle scattering function has been calculated by Stamm ${ }^{23}$ and it has been shown that characteristic maxima should appear which are not observed experimentally, ${ }^{8,24}$ however.

The question arises what kind of information can be obtained from the scattering data without introducing detailed structural models. The evaluation of the scattering data in a straight forward manner by Fourier-Bessel-transform is complicated by the formation of the "clusters," which we discussed aobve. The problem can be solved by use of the so-called "direct correlation function" $c(\underline{x})$ which was introduced by Ornstein-Zernike $(\mathrm{OZ})^{25}$ in the theory of critical opalescence. The meaning of the direct correlation function in connection with our problem is demonstrated in Figure 15.

The stem at 0 is directly correlated with the stems at 1 and 2 by means of the amorphous loops. But there is also a probability to meet another stem at point 1 because of a direct correlation between 1 and 2; if these stems were connected by a loop. So one obtains

$$
h(01)=c(0,1)+c(0,2) \otimes c(2,1)
$$

The convolution has to be repeated and leads in the classical case of critical opalescence finally to the

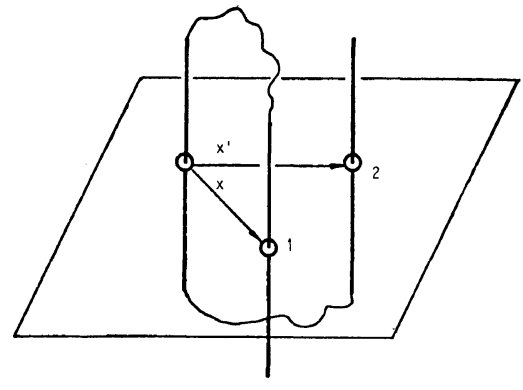

Figure 15. Application of the direct correlation function $c(x)$ to the problem of the crystallization of polymers.

$\mathrm{OZ}$ integral equation

$$
h(x)=c(x)+\rho_{0} \int c\left(x^{\prime}\right) h\left(x^{\prime}-x\right) \mathrm{d}^{2} x^{\prime}
$$

The treatment has to be modified for our problem, since one has to take into account the finite number of stems within a cluster. First we introduce the probability $\beta$ that the chain leaves a cluster and enters into another cluster. So starting with the first crystalline stem the probability of $n$ re-entries is given by

$$
p(n)=\alpha^{n} \beta
$$

with $\alpha=1-\beta$.

Secondly we repeat the convolution of eq 17 only $N$ times, where $N$ is the number of all crystalline stems of one molecule (molecular weight distribution is neglected). The result of this treatment is given by ${ }^{26,27}$ :

$$
H(q)=\frac{2 \alpha C(q)}{1-\alpha C(q)}\left[1-\frac{1}{N} \frac{1-(\alpha C(q))^{N}}{1-\alpha C(q)}\right]
$$

where $C(q)$ is the Fourier-Bessel transform of $c(x)$. It is normalized by

$$
C(q) \underset{q \rightarrow 0}{\longrightarrow} 1
$$

Therefore the expectation value for the average number $N_{\mathrm{c}}$ of stems per cluster in the limit $N \rightarrow \infty$ is given by

$$
\left\langle N_{\mathrm{c}}-1\right\rangle=2 \frac{\alpha}{1-\alpha} \quad \text { for } N \rightarrow \infty
$$

Equation 20 can be used to calculate the transform $C(q)$ of the direct correlation function $c(x)$ from the measured overall correlation function $H(q)$. If $C(q)$ is known the correlation function $c(x)$ can 
be obtained by a straight forward method, at least in principle.

With this method there are three major problems involved. i) The treatment so far does not take into account the crystal lattice. ii) It also neglects the cases where one lattice point in the basal plane of the lamella is already occupied by a crystalline stem of the same molecule (excluded volume effect) and iii) the back transformation $C(q)$ into $c(\underline{x})$ is truncated since for experimental reasons the available $q$ range is limited.

In order to check the effect of these three limitations Monte-Carlo calculations were performed. ${ }^{27}$ For several initial distribution functions $c(x)$, stem clusters were generated taking into account the crystal structure of poly(ethylene oxide). Starting from the first stem with a probability $(1-\beta)$ the next lattice point was chosen at the crystal basal plane shown in Figure 16. The proceeding was repeated taking into account the a priori distribution function $c(x)$. If the chosen lattice point is already occupied by a previous stem a new trial was made. If more than 10 trials had no success, this cluster was no longer taken into account and a new cluster was generated. After repeating this process 1000 times an averaged scattering function $H_{\mathrm{MC}}(q)$ is obtained as well as an "experimental" direct correlation function $C_{\mathrm{MC}}(q)$, which now includes the excluded volume effect. As indicated in Figure 16 two different types of clusters were generated, once with a one-dimensional arrangement of stems and secondly with rotational symmetry.

In order to test eq $20 H_{\mathrm{MC}}(q)$ was now considered

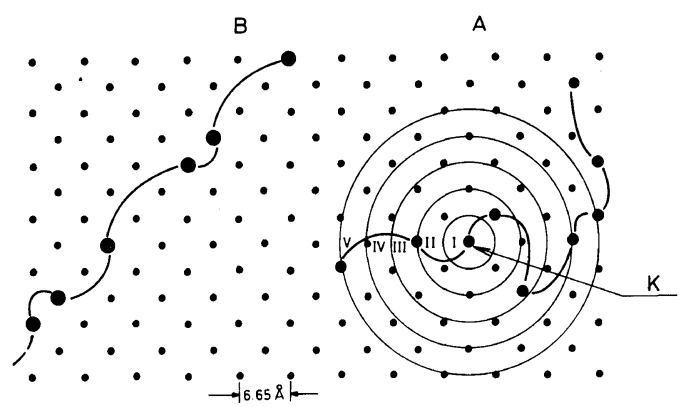

Figure 16. Arrangements of crystalline stems in the PEO-lattice for calculating the scattering functions of Monte-Carlo generated clusters. A) Rotational symmetry of the a priori direct correlation function $c(x)$; B) One dimensional arrangements of stems. as an experimentally obtained scattering curve and $C(q)$ was calculated according to eq 20 . In Figure 17 these values are compared with the "experimental" correlation function $C_{\mathrm{MC}}(q)$. For the first two cases, that means for a direct correlation function $c(x)$ which either continuously declines or which exhibits a maximum, the agreement between the analytically calculated $C(q)$ and the "real" $C_{\mathrm{MC}}(q)$ is very satisfactory. Especially it may be noticed, that two

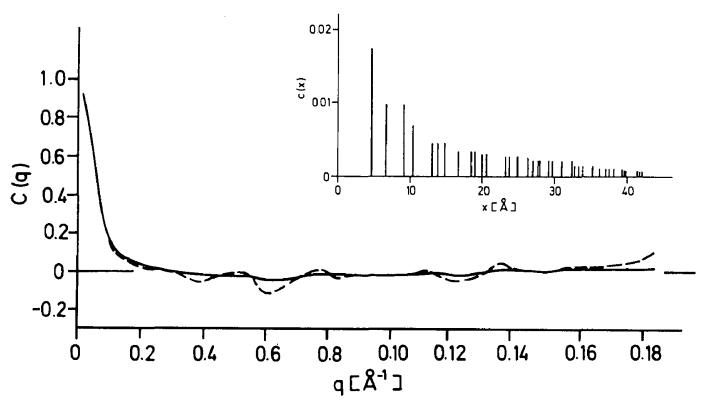

(a)

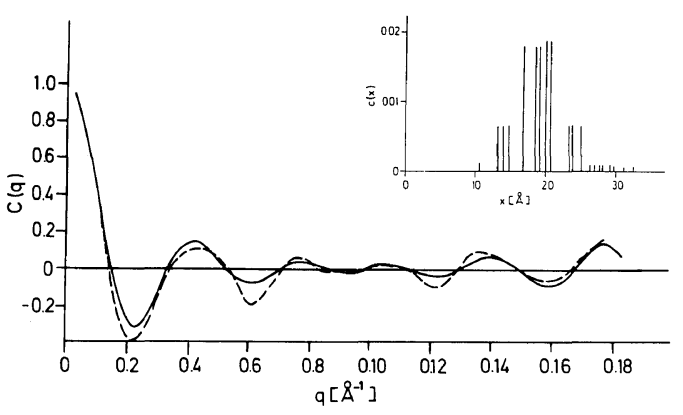

(b)

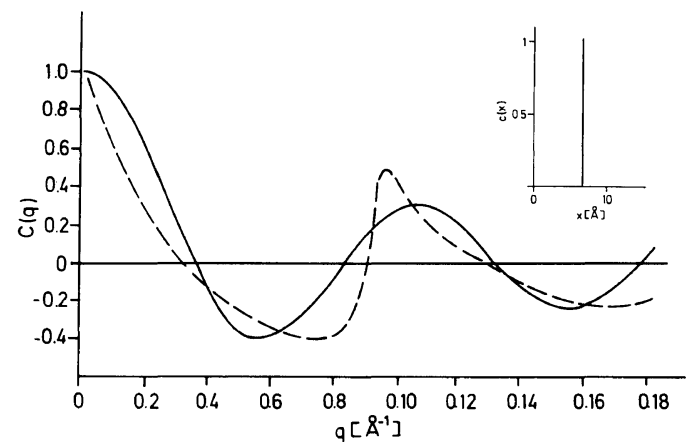

(c)

Figure 17. Fourier-Bessel transforms $C(q)$ of the direct correlation function $c(x) .-, C_{\mathrm{MC}}(q)$ as generated by Monte-Carlo method; ---, $C(q)$ as analytically calculated from $H_{\mathrm{MC}}(q)$ by means of eq 20. (a), (b), and (c), three different $c(x)$ shown in the insert. 
important features are well reproduced, which are interesting from the experimental point of view. These are the $q_{0}$-values for $C\left(q_{0}\right)=0$ and the depth of the first minimum. The third case in Figure 16 represents adjacent re-entry along the (100) plane. It is not surprising that with such a strong correlation eq 20 fails to some extent.

From a rather large number of Monte-Carlo experiments using varioús direct correlation functions $c(x)$ the general conclusion can be drawn, ${ }^{27}$ that $C(q)$ can be evaluated from the measured $H(q)$ by means of eq 20 in a good approximation, if $c(x)$ is not just a $\delta$-function. This result was obtained independently of assumptions about a twodimensional or a one-dimensional arrangement of crystalline stem centers.

The second problem which has been studied by Monte-Carlo experiments is the effect of truncation on the Fourier-Bessel transformation of $C(q)$. The direct correlation function $c(x)$ is obtained from

$$
c(x)=\lim _{q_{m} \rightarrow \infty} \int_{0}^{q_{m}} J(q) J_{0}(q x) 2 \pi q \mathrm{~d} q
$$

but the upper integration limit $q_{\mathrm{m}}$ is finite for experimental reasons. Especially in the case of poly(ethylene oxide) the truncation effect is rather serious for the following reason. The crystal structure has a repeating unit in chain direction of $c=$ $19.48 \AA$ corresponding to a layer line distance of $0.64 \AA^{-1}$ in the reciprocal space. The diffuse background scattering due to the $\mathrm{H}$ - and $\mathrm{D}$-chain mixture is repeated on each layer line. Therefore the measured neutron intensity at $q$-values $q>0.64 \AA$ is the sum of the intensities from the zero layer line and the first layer line. In principle these contributions can be separated, but in practice the scattering of the experimental data is too large for that purpose. So the transformation of eq 23 is restricted to $q_{\mathrm{m}} \sim 0.6 \AA^{-1}$. The situation would be more favourable for the case of polyethylene, of course, where $c=2.5 \AA$.

In Figure 18 the effects of the truncation error are demonstrated for two cases. $W(x)$ gives the probability for distances $r$ in the PEO lattice as they appear in the "real" Monte-Carlo generated clusters. $W_{\mathrm{tr}}(x)$ is the result of the back transformation of $C(q)$ which was obtained from the "experimental" scattering curve $H_{\mathrm{MC}}(q)$ by means of eq 20. For the back transformation $H_{\mathrm{MC}}(q)$ was truncated at $q=0.6 \AA^{-1}$.

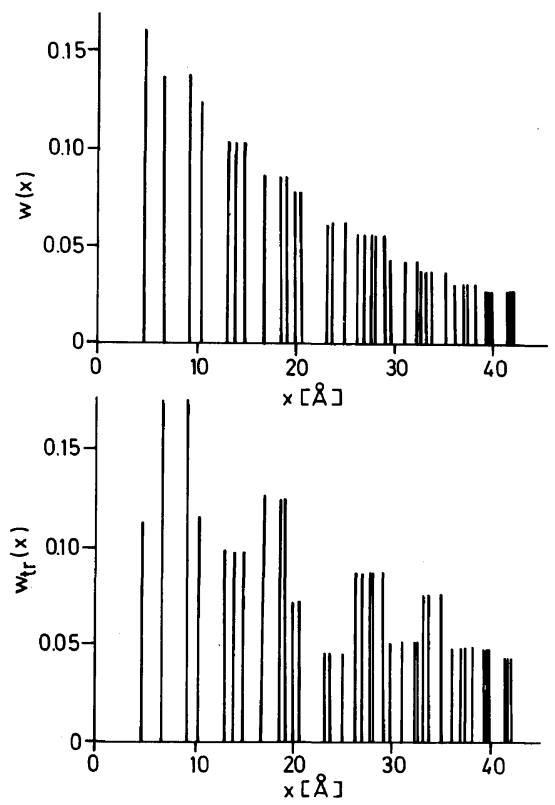

(a)

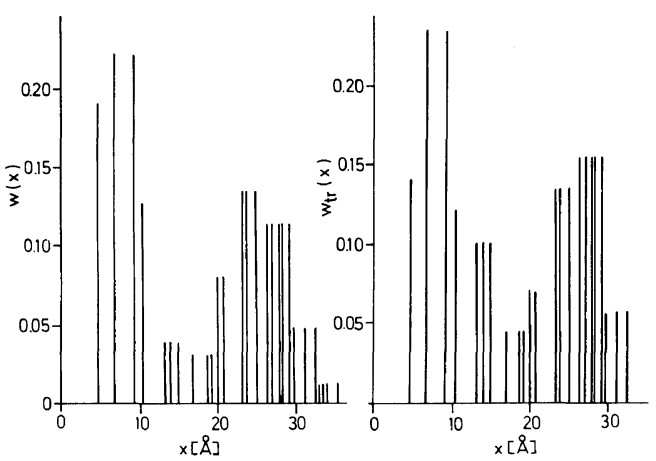

(b)

Figure 18. Probability distribution of stems within the clusters. $W(x)$ as generated by the Monte-Carlo method. $W_{\mathrm{tr}}(x)$ as calculated from the "experimental" scattering curve $H_{\mathrm{MC}}(q)$. Truncation of $H_{\mathrm{MC}}(q)$ at $q_{\mathrm{m}}=0.6 \AA^{-1}$.

The agreement is satisfying if one takes into account that only a rather limited $q$-range was available. Similar results were found for other distributions. ${ }^{27} \mathrm{We}$ therefore concluded that the method applied above can also be used for the experimental neutron scattering data. The procedure was the following: By means of eq 10 the stem correlation function $H(q)$ was obtained from the experimental data $J(q)$. Then $C(q)$ was calculated from eq 20 and finally the desired direct correlation function $c(x)$ 

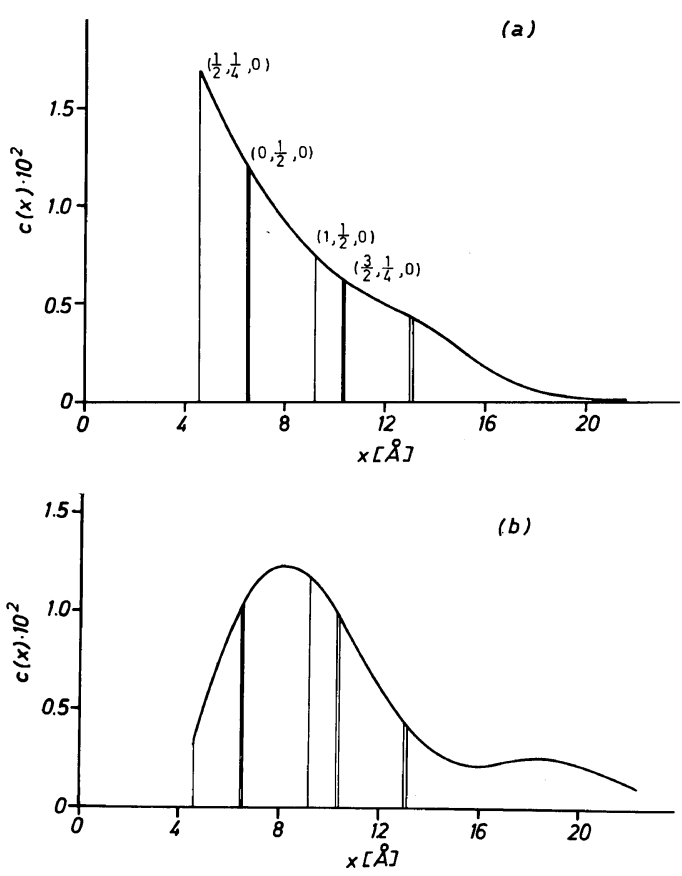

Figure 19. Direct correlation function $c(x)$ in crystalline PEO, $M_{w}=125,000$. (Probability density of subsequent crystalline stems belonging to the same molecule.) (a) Quickly crystallized at $T_{\mathrm{c}}=40^{\circ} \mathrm{C}(L=300 \AA)$; (b) Slowly cooled down $(L=430 \AA)$.

was obtained by the Fourier-Bessel transformation of eq 23.

The results for two samples of poly(ethylene oxide) $\left(M_{w}=125,000\right)$ crystallized under different conditions are plotted in Figure 19. The direct correlation functions $c(x)$, giving the probability densities for the location of the subsequent crystalline stem look very different for the two cases. For the quickly crystallized sample a continuously decreasing probability density is found and adjacent re-entry along (120) is most probable. In contrast the slowly crystallized sample with a large long spacing of $430 \AA$ exhibits a maximum at about $8 \AA$. This may be due to a mixture of preferred adjacent re-entry along (100) and (160) which corresponds to $6.50 \AA$ and $10.4 \AA$. It also may be possible that growth occurs along (120) but then only the neighbour after next is occupied $(9.2 \AA)$.

Single crystals (of PEO) crystallized at low undercooling exhibits growth faces parallel to (100) and (140). ${ }^{28}$ This may be in agreement with the preferred adjacent re-entry given above, if one neglects the small angle $\left(\sim 8^{\circ}\right)$ between (140) and (160).

\section{REFERENCES}

1. I. G. Voigt-Martin and L. Mandelkern, J. Polym. Sci., Polym. Phys. Ed., 19, 1769 (1981).

2. G. Kanig, Progr. Colloid Polym. Sci., 57, 176 (1975).

3. P. J. Flory, Pure Appl. Chem., 56, 305 (1984).

3a. D. Y. Yoon and P. J. Flory, Faraday Discuss. Chem. Soc., 68, 288 (1979).

4. See, for example, "Organization of Macromolecules in the Condensed Phase," Faraday Discuss. Chem. Soc., 68 (1979).

5. J. D. Hoffman, C. M. Guttman, and E. A. DiMarzio, Faraday Discuss., 68, 177 (1979).

6. D. G. H. Ballard and J. Schelten, "Developments in Polymer Characterization," Vol. 2, J. V. Dawkins, Ed., Appl. Science, London, 1980.

7. E. W. Fischer, Pure Appl. Chem., 50, 1319 (1978).

8. M. Stamm, E. W. Fischer, M. Dettenmaier, and P. Convert, Faraday Discuss. Chem. Soc., 68, 263 (1979).

9. J. Schelten, D. G. H. Ballard, G. D. Wignall, G. Longmann, and W. Schmatz, Polymer, 17, 751 (1976).

10. D. G. H. Ballard, P. Cheshire, G. W. Longman, and J. Schelten, Polymer, 19, 379 (1978).

11. J.-M. Guenet and C. Picot, Macromolecules, 16, 205 (1983).

12. J. Kugler, U. Struth, R. Born, E. W. Fischer, and K. Hahn, in preparation.

13. J. D. Hoffman, Polymer, 24, 3 (1983).

14. E. W. Fischer, M. Stamm, M. Dettenmaier, and P. Herchenröder, Polym. Prepr., Am. Chem. Soc., Div. Polym. Chem., 20, 219 (1979).

15. D. M. Sadler, R. Harris, and H. H. Wills, J. Polym. Sci., Polym. Phys. Ed., 20, 561 (1982).

16. E. W. Fischer, Faraday Discuss. Chem. Soc., 68, 425 (1979).

17. C. M. Guttman, J. D. Hoffman, and E. A. DiMarzio, Faraday Discuss. Chem. Soc., 68, 297 (1979).

18. E. W. Fischer, K. Hahn, J. Kugler, U. Struth, R. Born, and M. Stamm, J. Polym. Sci., Polym. Phys. $E d$., in press.

19. D. G. H. Ballard, G. W. Longman, T. L. Crowley, A. Cunningham, and J. Schelten, Polymer, 20, 399 (1979); D. G. H. Ballard, A. N. Burgess, A. Nevin, P. Cheshire, G. W. Longman, and J. Schelten, Macromolecules, 13, 677 (1980).

20. J. M. Guenet and C. Picot, Polymer, 20, 1483 (1979).

21. D. G. H. Ballard, P. Cheshire, E. Janke, A. Nevin, and J. Schelten, Polymer, 23, 1875 (1982).

22. E. W. Fischer, "IUPAC Macromolecules," $\mathrm{H}$. Benoit and P. Rempp, Ed., Pergamon, London, 1982.

23. M. Stamm, J. Polym. Sci., Polym. Phys. Ed., 20, 235 (1982). 


\section{E. W. FISCHER}

24. G. D. Wignall, L. Mandelkern, C. Edwards, and M. Glotin, J. Polym. Sci., Polym. Phys. Ed., 20, 245 (1982)

25. L. S. Ornstein and F. Zernike, Proc. Acad. Amsterdam, 17, 793 (1914).

26. M. Dettenmaier, E. W. Fischer, and M. Stamm,
Colloid Polym. Sci., 258, 343 (1980).

27. A. Sariban, K. Hahn, E. W. Fischer, and M. G. Brereton, in preparation.

28. A. J. Kovacs, C. Straupe, and A. Gonthier, J. Polym. Sci., Polym. Symp., 59, 31 (1977). 